\title{
La Actitud de Investigadores sobre la Cooperación Tecnocientífica entre Universidades y Sector Productivo: Un Estudio Comparativo
}

\author{
Juan Carlos Pérez-Morán ${ }^{1 *}$, Marcela Morales Páez ${ }^{1}$,Brando Bernal-Baldenebro ${ }^{1}$
}

Resumen: El propósito del presente estudio es medir la actitud hacia la cooperación tecno-científica entre Instituciones de Educación Superior y Empresas del Sector Productivo de profesores-investigadores, así como analizar las diferencias por sexo, edad, y nivel en el Sistema Nacional de Investigadores. Se utilizó una muestra no aleatoria por conveniencia de 182 p-i (50 mujeres y 132 hombres) quienes contestaron el Cuestionario para medir la percepción de la cooperación tecno-científica entre las Instituciones de Educación Superior y el Sector Productivo. Entre los hallazgos, se destacan valores de diferencia no significativa $(p>.05)$, y tamaño de efecto chico $\left(\eta^{2}=.01\right)$ entre los grupos.

Palabras clave: colaboración universidades-industria; investigadores universitarios, actitud; sexo; edad; ANOVA; invarianza

Abstract: Title: Researchers' Attitude of Techno-Scientific Cooperation Between Universities and The Productive Sector: A Comparative Study The purpose of this study is to measure the attitude towards techno-scientific cooperation between universities and the productive sector of professors-researchers and to analyze the differences by gender, age, and level in the National System of Researchers. A non-random convenience sample of $182 \mathrm{p}$-i (50 women and $132 \mathrm{men}$ ) from universities and research centers located in the Northwest region of Mexico was used who answered the Questionnaire to measure the perception of techno-scientific cooperation between Higher Education Institutions and the Productive Sector. Among the findings, values of non-significant difference $(p>.05)$ and small effect size $\left(\eta^{2}=.01\right)$ between groups.

Keywords: university-industry collaboration; university researchers; attitude; gender; age; SNI; ANOVA; invariance

Submitted: August $18^{\text {th }}, 2021 /$ Approved: November $3^{\text {rd }}, 2021$

\section{Antecedentes del estudio}

Diversas investigaciones destacan que, desde hace ya varias décadas, se estableció una convergencia entre Instituciones de Educación Superior (IES) y Empresas del Sector Productivo (ESP) hacia los procesos de generación y comercialización del conocimiento, así como el importante papel de las universidades como promotoras del desarrollo tecnológico y la innovación (Etzkowitz, 2003; Fichter y Tiemann, 2018; García-Galván, Lindquist y Morales, 2021). En este contexto, es fundamental conocer cuáles son las características de esta cooperación bidireccional, denominada cooperación tecno-científica por Morales (2019); en especial, la actitud de los profesores-investigadores (p-i) hacia la cooperación tecno-científica, así como las diferencias entre grupos con características distintivas; ya que ellos son los actores principales de esta interacción.

A este respecto, es importante destacar que la colaboración entre IES y su entorno es de naturaleza diversa. En el ámbito académico mexicano, dicha cooperación se relaciona con términos como "extensión de la docencia y la investigación", "vinculación con otros actores", y "transferencia de tecnología y de los resultados de investigación". Morales (2019) acota esta dispersión conceptual y propone el uso del término "cooperación tecno-científica", a partir de los fundamentos del Institucionalismo contemporáneo integrado, ya que dicho término permite unificar y clarificar la relación bidireccional IES-ESP basada en la producción de conocimiento.
En la práctica, el estudio de la cooperación IES-ESP se ha dado desde diferentes perspectivas teórico-epistemológicas: los Sistemas de Innovación, el Modo 2 de Producción del Conocimiento, el Modelo de la Triple Hélice, y el Institucionalismo Contemporáneo Integrado. El enfoque de los Sistemas de Innovación, originado en la década de 1980, se generó como marco de referencia para el diseño de políticas públicas de innovación, sin embargo, se ha establecido con fuerza en los contextos académicos. A pesar de que en sus inicios el énfasis se dió en el ámbito nacional, la generación de sistemas de innovación sectoriales y regionales hace necesaria la utilización del concepto de "sistema de innovación", con sus variantes nacional, sectorial y regional (Edquist, 2001, como se citó en Morales, 2019). Por otra parte, el Modo 2 es una nueva forma de producir conocimiento que incluye aspectos adicionales al tipo de conocimiento que es producido, por ejemplo, el contexto en el que se produce, los agentes que lo producen, y la organización y control de la calidad del mismo. Los autores destacaron el hecho de que el conocimiento especializado representa una ventaja competitiva para las empresas, las cuales, al no poder solventar los altos costos de la producción del mismo, se ven en la necesidad de establecer relaciones de cooperación con universidades, gobiernos e, inclusive, con otras empresas (Gibbons et al., 1997, como se citaron en Morales, 2019).

El Modelo de la Triple Hélice de relaciones entre la universidad, la industria y el gobierno trasciende los modelos anteriores de relaciones institucionales, como el laissez-faire o el socialista, en los

(1) Universidad Autónoma de Baja California, Instituto de Innovación y Desarrollo Educativo, Baja California México

${ }^{*}$ Corresponding author: juan.carlos.perez.moran86@uabc.edu.mx

ISSN: 0718-2724. (http://jotmi.org)

Journal of Technology Management \& Innovation @ Universidad Alberto Hurtado, Facultad de Economía y Negocios. 
cuales predominaba la empresa o el gobierno, con el sector académico en un papel secundario. Así, la dinámica de la sociedad ha cambiado desde una estructura con fronteras rígidas entre las diversas esferas institucionales, hacia un sistema más flexible, con cada actor realizando actividades antes restringidas a los otros (Etzkowitz et al., 2000). Por último, desde la perspectiva del Institucionalismo Contemporáneo Integrado, destaca el estudio de García-Galván (2008), quien utilizó este enfoque integrador al incorporar las teorías de los costos de transacción, de los recursos y capacidades, así como la teoría evolucionista, para dar cuenta de un análisis más detallado de la colaboración empresa-universidad.

Con respecto a las variables analizadas en investigaciones relacionadas con la cooperación tecnocientífica, Bozeman et al. (2013) y Perkmann et al. (2013) encuentran que estas se pueden organizar en las dimensiones individual, colaborativa, organizativa o institucional, y de impacto. La dimensión individual se centra en las características personales (p. ej. sexo, edad, profesión, experiencia) de los colaboradores en actividades de cooperación tecno-científica. La dimensión colaborativa se basa en los procesos de cooperación entre agentes de IES y ESP (p. ej. estilo de gestión, apertura, roles en la organización). La dimensión organizativa o institucional se centra en las características internas (p. ej. número de colaboradores, disciplina, tipo de organización) y externas (p. ej. políticas públicas, competencia, regulación gubernamental) de la organización en la cooperación tecno-científica. Por último, la dimensión de impacto se refiere a la repercusión que las actividades y productos de la cooperación tecno-científica (p. ej. productividad científica y comercial, preferencia en investigación aplicada, comportamiento colaborativo) tienen en académicos, IES y otras partes interesadas.

En particular, Morales (2019) llevó a cabo un estudio enfocado a clarificar y profundizar en las características y condiciones en que se llevan a cabo las actividades de cooperación entre IES y ESP de Baja California. Como parte de esta investigación se diseñó y aplicó el Cuestionario para medir la percepción de la cooperación tecnocientífica entre IES y ESP (CP-COOPTEC). Este instrumento permite recuperar información acerca de las actividades de investigación básica y aplicada, encaminadas a generar vínculos entre diversas organizaciones, a partir del reconocimiento de los procesos innovadores desde su origen hasta su aplicación en el ámbito productivo. Para el desarrollo de las tablas de especificaciones del CP-COOPTEC se trabajó bajo la supervisión de un experto en materia de cooperación tecno-científica, desde el enfoque teórico del Institucionalismo Contemporáneo Integrado. De inicio, el CP-COOPTEC estuvo integrado por 70 ítems, organizados en tres secciones que incluyeron dos variables sociodemográficas, cinco laborales, y 17 asociadas a la cooperación tecno-científica; así como por una escala de percepción con 6 subescalas y 46 ítems. La subescala para medir el Componente cognitivo de la actitud hacia la cooperación tecno-científica (parte 1) incluyó cuatro ítems; la subescala denominada Componente afectivo de la actitud hacia la cooperación tecno-científica constó de ocho ítems; la subescala Percepción sobre los apoyos institucionales hacia la cooperación tecno-científica, de tres; la subescala Componente cognitivo de la actitud hacia la cooperación tecno-científica (parte II), de 15; la subescala denominada Percepción sobre la infraestructura y gestión de la cooperación tecno-científica, de siete; y la subescala Percepción del marco institucional de la cooperación tecno-científica, de nueve ítems.

Posteriormente, en el estudio de Morales y García-Galván (2020) se determinó el nivel de asociación entre algunas de las variables exploradas en el del CP-COOPTEC mediante la aplicación de la prueba estadística Chi-cuadrado. Por su parte, Morales y Rodríguez (2021) presentaron la obtención de evidencias de validez de constructo del aspecto de estructura interna y confiabilidad del instrumento, mediante el cálculo del Alpha de Cronbach, siendo la confiabilidad global de todas las subescalas de (0.918).

\section{Características que influyen en la cooperación tecno-científica}

La literatura revisada señala distintos factores asociados a la realización de las actividades de la cooperación tecno-científica (Bozeman et al., 2014; Perkmann et al., 2013). Bozeman et al. (2014), en su marco organizativo de colaboración entre investigación en las universidades y en el ámbito académico empresarial, distinguen la importancia de los atributos del colaborador, la colaboración y la organización. El primero hace referencia a las características demográficas de los investigadores, tales como sexo, edad, experiencia, nacionalidad, entre otros; el segundo, se refiere a los procesos de colaboración en la investigación y composición de la colaboración, y cómo es que los atributos entre grupos afectan a las actividades de cooperación tecnocientífica; por último, el tercero se refiere a los atributos organizativos e institucionales a un nivel macro. En especial, la importancia de los atributos del colaborador se debe a que, en ocasiones, estas diferencias individuales afectan la interacción del investigador con organizaciones externas a la universidad en donde labora (Craig y Ponomariov, 2009; Bozeman et al. 2014).

Con respecto a la influencia del sexo en la realización de actividades de cooperación tecno-científica, se reporta que las mujeres participan en menor medida en actividades científicas y de cooperación con la industria (Bozeman et al. 2014; Ding et al., 2006; Perkmann et al. 2013; Tartari y Salter, 2015). Uno de los trabajos más representativos sobre el tema es el de Ding et al. (2006); en esta investigación se analizaron los datos de 4,227 bio-científicos y se encontró que las mujeres patentan un 0.40 en relación a los hombres, tienen pocos contactos en la industria y muchas de ellas se sienten en desventaja frente a los investigadores hombres debido a su escasa experiencia. Asimismo, en un estudio más reciente, Tartari y Salter (2015) encuestaron a 2,194 investigadores británicos y encontraron que las mujeres se encuentran involucradas en menor medida en la colaboración universidadindustria en relación con los hombres, así como que estas diferencias se acentúan en campos con menos mujeres y, en el caso del contexto local, la presencia de mujeres no conlleva a una mayor participación universidad-industria por parte de las mismas.

No obstante, hay estudios en donde se reporta que esta tendencia no es universal. Por ejemplo, Bozeman and Gaughan (2011), al controlar las variables de antigüedad, edad, situación familiar y disciplina, en 1,700 investigadores sustentantes de la U.S. National Survey of 
Academic Scientists, encontraron que las mujeres llevan a cabo ligeramente más actividades de colaboración universidad-industria en relación a los hombres. De la misma manera, Haeussler y Colyvas (2011) aplicaron un cuestionario a 629 y a 1,665 bio-científicos alemanes y británicos, respectivamente, en donde encontraron que el sexo no es un factor determinante para realizar actividades de consultoría y el registro de patentes. Lo anterior puede ser debido a que la brecha de género está disminuyendo (Ding et al., 2006) y que la presencia de mujeres aumenta la participación de las mismas en actividades de cooperación universidad-industria (Tartari y Salter, 2015).

Con relación a la experiencia académica o profesional, se reporta que los investigadores con mayor experiencia y antigüedad se comprometen en mayor medida en la realización de actividades de cooperación tecno-científica (Perkmann et al., 2013). Los investigadores con mayor experiencia suelen tener redes de investigación más amplias y consolidadas en relación a aquellos que no cuentan con tanta experiencia (Haeussler y Colyvas, 2011; Perkmann et al., 2013; Stuart y Ding, 2006). Stuart y Ding (2006) mencionan dos razones potenciales por las cuales se puede dar esta relación: (a) los investigadores con mayor experiencia tienen la capacidad y reputación de atraer recursos por parte del sector privado; y (b) los investigadores con mayor experiencia académica se intimidan menos por la amenaza de pérdida de reputación por realizar actividades de cooperación tecno-científica. Asimismo, D’Este y Patel (2007) encontraron que los investigadores con mayor experiencia profesional cuentan con comportamientos asociados a la realización de actividades de cooperación tecno-científica.

Referente a la influencia de la edad se reportan resultados y conclusiones mixtas (Bozeman et al., 2014; Perkmann et al., 2013). Por una parte, Haeussler y Colyvas (2011) exponen una relación positiva entre la edad y cooperación tecno-científica; encontraron que entre los investigadores alemanes y británicos encuestados, a mayor edad mayor es el compromiso para realizar actividades de cooperación tecnocientífica. Por otra parte, D’Este y Patel (2007) encuestaron a 1,528 investigadores británicos, y reportan que, al contrario de Haeussler y Colyvas (2011), los investigadores jóvenes son aquellos que se comprometen en mayor medida a las actividades de cooperación tecnocientífica. De manera similar, Giuliani et al. (2010) entrevistaron a 135 investigadores de distintos países (40 chilenos; 42 sudafricanos; 53 italianos) y encontraron que los investigadores jóvenes son más propensos a colaborar con la industria en relación a los investigadores de mayor edad. Otro punto es que la edad sea un factor irrelevante para la cooperación tecno-científica, Craig y Ponomariov (2009) analizaron los datos de una encuesta de nivel nacional aplicada a 1,643 científicos estadounidenses y encontraron que la edad se relacionaba de manera no significativa en la mayoría de las variables de cooperación tecno-científica.

Dado a lo anterior, el propósito del presente estudio fue profundizar en la obtención de evidencias de invarianza factorial y en el impacto que las características personales de los p-i tienen sobre la percepción de actividades de cooperación tecno-científica. Las evidencias de invarianza factorial se obtuvieron mediante la aplicación de un Análisis Factorial Multigrupo (AFCMG); y se verificó el impacto de las características personales sobre percepción de actividades de cooperación tecno-científica a través de análisis de varianza (ANOVA) y cálculo del coeficiente eta cuadrada $\left(\eta^{2}\right)$.

\section{Método}

\section{Participantes}

Se utilizó la base de datos del estudio de Morales (2019), en donde se encuestó a 184 profesores-investigadores ( $\mathrm{p}$-i) mexicanos de Instituciones de Educación Superior (IES) del estado de Baja California, México. Todos los participantes pertenecen al padrón del Sistema Nacional de Investigadores (SNI) del Consejo Nacional de Ciencia y Tecnología (CONACYT), y señalan haber participado en actividades de cooperación tecno-científica en algún momento de su vida profesional. Para los propósitos del presente estudio se trabajó con una muestra de $182 \mathrm{p}$-i resultado de eliminar dos casos atípicos. De la muestra total, $132 \mathrm{p}-\mathrm{i}$ son hombres $(72.5 \%)$ y 50 (27.5\%) son mujeres. Porcentajes similares en cuanto a la distribución por sexo de p-i en IES se reportan en estudios de este tipo (pe. Bozeman and Gaughan, 2011; Ding et al., 2006; Haeussler y Colyvas, 2011; Tartari y Salter, 2015). Con referencia a la edad de los p-i encuestados, 22 se encuentran en el grupo de edad de 35 años o menos (12.1\%); 39 en el grupo de 36 a 40 años (21.4\%); 33, en el grupo de 41 a 45 años (18.1\%); 14, en el grupo de 46 a 50 años (7.7\%); y 74, en el grupo de edad de 50 años o más (40.7\%).

\section{Instrumento}

Se utilizó la Escala de Actitud hacia la Cooperación Tecno-científica entre IES y ESP (EA-COOPTEC v0.1), ajustada con base en el estudio realizado por Pérez-Morán et al. (2021). Dicha escala es parte del Cuestionario para medir la Percepción de la Cooperación Tecnocientífica entre IES y ESP (CP-COOPTEC) desarrollado por Morales (2019). La EA-COOPTEC v0.1 mide la percepción de los encuestados en relación a las actividades de vinculación entre IES y ESP (cooperación tecno-científica) por medio de cuatro ámbitos (factores): (a) beneficios comunitarios de la cooperación tecno-científica $(\mathrm{k}=10)$, (b) beneficios personales de la cooperación tecno-científica $(\mathrm{k}=7)$, (c) responsabilidad institucional para la cooperación tecno-científica $(\mathrm{k}=9)$, y (d) regulación y normatividad de las actividades de cooperación tecno-científica $(\mathrm{k}=3)$. Dicho instrumento, se compone por 29 ítems de tipo Likert con cuatro categorías en escala ordinal ( $T o$ talmente en desacuerdo $=1$, En desacuerdo $=2$, De acuerdo $=3$, Totalmente de acuerdo $=4$ ). Asimismo, Pérez-Moran et al. (2021) reportaron evidencias de la validez de constructo del aspecto de la estructura interna de la EA-COOPTEC v0.1; los índices de confiabilidad interna de las subescalas presentaron valores aceptables ( $\alpha$ de .83 hasta $.89 ; \rho$ de .83 hasta .89 ; y $\omega$ de .73 hasta .90$)$ y valores adecuados de ajuste $\left(\chi^{2}\right.$ $=558.080, \mathrm{gl}=366, \mathrm{p}<0.00, \mathrm{CFI}=0.92, \mathrm{TLI}=0.91, \mathrm{GFI}=0.82, \mathrm{NFI}$ $=0.81$, RMSEA $=0.05[$ IC 95\% $=0.04 ; 0.06]$, SRMR $=0.06$ ). Además, en el mismo estudio, se obtuvieron evidencias de invarianza factorial por sexo y edad; encontrándose evidencia de ajuste adecuado en el modelo de invarianza factorial estricto para grupos de distinto sexo y evidencias de invarianza fuerte para grupos de distintas edades. Con ello, se puede asumir que tanto los p-i hombres y mujeres, así como los p-i jóvenes y de mayor edad entienden de la misma manera el constructo medido por la EA-COOPTEC v0.1. 


\section{Análisis de datos}

Se pusieron a prueba tres hipótesis de investigación, con base en la información obtenida de la revisión de la literatura. Para la primera hipótesis $\left(\mathrm{H}_{1}\right)$ se propone que los $\mathrm{p}$-i hombres en promedio tienen mayor predisposición a realizar actividades de cooperación tecno-científica entre IES y ESP que las p-i mujeres. Para la segunda hipótesis $\left(\mathrm{H}_{2}\right)$, se plantea que los p-i con nivel más alto en el SNI, en comparación con los p-i con nivel más bajo, cuentan en promedio con una actitud más favorable para realizar actividades de cooperación tecno-científica entre IES y ESP. Por su parte, para la tercera hipótesis $\left(\mathrm{H}_{3}\right)$, se considera que los investigadores de mayor edad, en comparación con los de menor edad, cuentan con una actitud más favorable para realizar actividades de cooperación tecno-científica entre IES y ESP.

Con el fin de evaluar las hipótesis del estudio, se definieron los grupos de comparación y, subsecuentemente, se analizaron sus diferencias en cuanto a su predisposición para realizar actividades de cooperación tecno-científica entre IES y ESP. Para la $\mathrm{H}_{1}$ los participantes fueron divididos en grupos de hombres y mujeres. Para la $\mathrm{H}_{2}$ se consideraron los tipos de distinción en el SNI del CONACyT (Art. 28, Diario Oficial de la Federación, 2021) organizando en un primer grupo a los p-i con la distinción de Candidato a investigador nacional y de Investigador nacional Nivel 1, y en el segundo grupo, a los p-i con distinción de Investigador nacional Nivel 2 y Nivel 3. Para los propósitos del estudio, y con base en la revisión de la literatura, se consideró pertinente el uso de las distinciones del SNI establecidas por el CONACyT como un indicador relevante y preciso de la experiencia y capacidad de los p-i para generar investigación de calidad (D'Este y Patel, 2007; Haeussler y Colyvas, 2011; Morales, 2009; Perkmann et al., 2013; Puerta-Sierra y Jasso, 2020; Stuart y Ding, 2006). Por último, para la $\mathrm{H}_{3}$ se dividió a los participantes en dos grupos de edad, el primero conformado por aquellos p-i con 45 años o menos, y el segundo conformado por p-i mayores de 45 años. Asimismo, se establecieron como variables independientes el sexo, la edad y el nivel en el SNI de los p-i encuestados; la variable dependiente para el estudio fue la puntuación total de la EA-COOPTEC v0.1.

Los análisis estadísticos para la obtención de evidencia de invarianza factorial y la comprobación de hipótesis se hicieron por medio del software RStudio en su versión 1.4, principalmente utilizando las paqueterías $d p l y r$, psych, lavaan y semTools, y con el programa IBM SPSS Statistics en su versión 23. Dichos análisis se dividieron en cuatro etapas: (1) obtención de estadísticos descriptivos, (2) análisis de supuestos de normalidad y confiabilidad, (3) medición de invarianza factorial en función del nivel de distinción en el SNI de los p-i, y (4) análisis de las diferencias entre grupos. En la primera etapa se obtuvo la media y desviación media de las puntuaciones de los sustentantes. En la segunda etapa se verificó el supuesto de normalidad de la muestra por medio de la prueba de Shapiro-Wilk; el criterio para aceptar dicho supuesto se estableció en la obtención de un valor del índice no significativo ( $\mathrm{p} \geq .05$; Creswell, 2012). Asimismo, se calcularon los índices alfa de Cronbach ( $\alpha$ ), alfa estandarizada Rho $(\rho)$ y el coeficiente Omega de McDonald ( $\omega$ ) como medidas de confiabilidad de la medición; el criterio para verificar dicho supuesto fue $\alpha \geq 0.70, \rho \geq$ 0.70 y $\omega \geq 0.80$ (Hair et al., 2019). Enseguida, se verificó el supuesto de invarianza factorial en función del sexo y nivel de distinción en el SNI de los p-i. Para ello se tomó la estructura de cuatro factores del EA-COOPTEC v0.1 propuesta por Pérez-Morán et al. (2021) y se consideraron las sugerencias de $\mathrm{Hu}$ y Bentler (1999) para el ajuste del modelo configuracional en la medición de la invarianza; como criterio se consideró un ajuste comparativo (CFI) $\geq .90$, residuo estandarizado cuadrático medio $(\mathrm{SRMR}) \leq .08$ y error cuadrático medio de aproximación (RMSEA) $\leq .08$. Consecutivamente, se aplicó el AFCMG, siguiendo las recomendaciones de Dimitrov (2010); los criterios para la aceptación de la invarianza factorial fue que la diferencia de chi-cuadrada $(\Delta \chi 2)$ entre los modelos anidados presentara valores no significativos ( $\mathrm{p} \geq .05)$. Por último, se verificó la diferencia entre grupos a través de un ANOVA de las puntuaciones medias de los grupos; el criterio para aceptar la diferencia entre grupos se estableció en la obtención de valores de diferencia significativa ( $\mathrm{p} \leq$ .05; Creswell, 2012). Asimismo, se siguieron las recomendaciones de Funder y Ozer (2019) para el cálculo e interpretación del tamaño del efecto por medio del coeficiente eta cuadrada $\left(\eta^{2}\right)$.

\section{Resultados}

\section{Análisis descriptivos y supuesto de normalidad}

La puntuación media y la Desviación Estándar (DE) global de la EA-COOPTEC v0.1 obtenidas en el estudio fueron de 85.98 y 11.31, respectivamente. Las puntuaciones medias de las cuatro subescalas fueron: $29.75(\mathrm{DE}=5.88)$ para la primera (beneficios comunitarios de la cooperación tecno-científica), 23.83 ( $\mathrm{DE}=3.27$ ) para la segunda (beneficios personales de la cooperación tecno-científica), 22.26 (DE = 5.09) para la tercera (responsabilidad institucional para la cooperación tecno-científica), y $10(\mathrm{DE}=1.65)$ para la cuarta (regulación y normatividad de las actividades de cooperación tecno-científica). Por su parte, la media y la desviación estándar en función de las variables independientes y de las subcategorías que las componen se pueden observar en la tabla 1. Nótese que, la mayor diferencia inmediata entre las puntuaciones medias de las subcategorías se encuentra entre la distinción como Candidato(a) a investigador(a) nacional y la distinción como Investigador(a) nacional Nivel 1. 
Tabla 1: Puntuación media y desviación estándar del índice general de la EA-COOPTEC v0.1 de acuerdo con el sexo, grupo de edad, y nivel en el SNI de los p-i

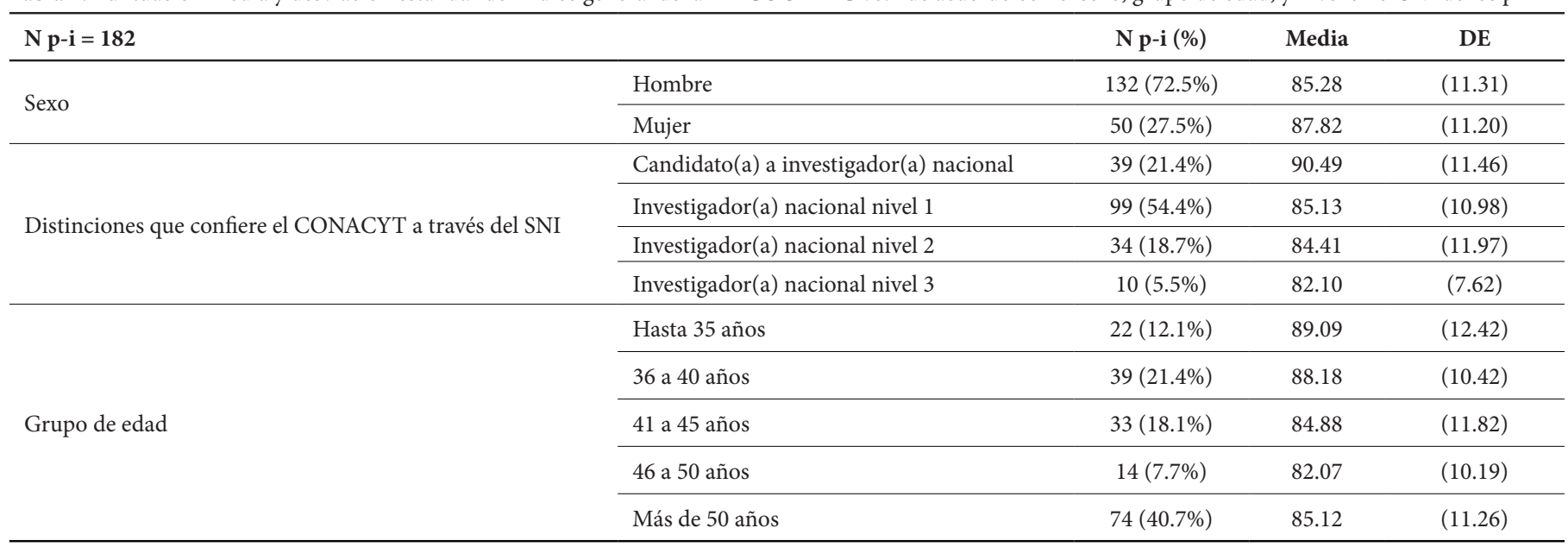

El análisis de confiabilidad arrojó índices Alpha de Cronbach global de .91, Alpha estandarizada de .91 y un coeficiente Omega de McDonald de .94. Estos resultados se consideran adecuados y similares a las investigaciones de Pérez-Moran et al. (2021) y Morales y Rodríguez (2021). Con relación al supuesto de normalidad en las puntuaciones, la prueba de Shapiro-Wilk arrojó un valor superior al criterio establecido para verificar el supuesto de normalidad ( $p=.24 ; g l=182)$, por lo que se considera que los puntajes de la prueba se distribuyen de manera normal.

\section{Invarianza factorial en función del nivel del SNI de los p-i}

Con el fin de verificar si los p-i participantes con distinto nivel de SNI conceptualizan de la misma manera el constructo a medir, se aplicó un AFCMG en donde se examina evidencia de invarianza factorial (ver Tabla 2). Los resultados muestran evidencia de ajuste moderado para el modelo configuracional $\left(\chi^{2}=896.96\right.$; $\mathrm{gl}=576$; $p<0.00$; $\mathrm{CFI}=0.873$; RMSEA $=0.078$ [IC 95\% $=0.062 ; 0.081]$ ). Asimismo, se encuentra evidencia para la aceptar el modelo débil y, por lo consiguiente, que las cargas factoriales de ambos grupos es la misma entre estos $\left(\Delta \chi^{2}=26.75 ; p=.22\right)$. Sin embargo, no se obtuvieron evidencias que sustenten un ajuste adecuado en el modelo de invarianza fuerte $\left(\Delta \chi^{2}=45.55 ; p=.00\right)$. Por lo que, siguiendo las recomendaciones encontradas en Dimitrov (2010), se liberaron los intercepto de los ítems PBC.02, PBC.05 y PBC.10 y de esta manera se obtuvieron valores adecuados para la aceptación de un modelo de invarianza fuerte parcial $\left(\Delta \chi^{2}=29.98 ; p=.06\right)$.

Tabla 2: Análisis de invarianza factorial en función del nivel del SNI de los p-i

\begin{tabular}{lccccccc}
\hline Modelo & $\boldsymbol{\chi}^{2}$ & $\boldsymbol{\Delta} \boldsymbol{\chi}^{2}$ & $\boldsymbol{g l}$ & $\boldsymbol{p}$ & CFI & $\Delta$ CFI & RMSEA \\
\hline Configuracional & 896.96 & - & 576 & - & .873 & .078 \\
\hline Débil & 920.66 & 23.700 & 598 & .36 & .872 & -.001 \\
\hline Fuerte & 967.21 & 45.549 & 620 & $.00^{* *}$ & .862 & -.01 & .077 \\
\hline Fuerte parcial & 949.60 & 29.937 & 617 & .067 & .868 & -.004 & .078 \\
\hline Estricto & 1030.45 & 80.853 & 646 & $8.7 \mathrm{e}-7^{* *}$ & .848 & -.014 & .081 \\
\hline
\end{tabular}

${ }^{*} p \leq .05 ;{ }^{* *} p \leq .01$

\section{Diferencias entre grupos}

Se aplicaron análisis estadísticos con el fin verificar si se presentaban diferencias entre grupos. En primer lugar, se verificaron las diferencias de los sustentantes en función del sexo. Los resultados del análisis permiten comprobar diferencias no significativas entre sexos (F $=1.84 ; p=.177 ; \eta^{2}=.01$ ), por lo que se infiere que ambos grupos cuentan con una actitud similar para realizar actividades de cooperación tecno-científica entre IES y ESP. En segundo lugar, se revisaron las diferencias en función de nivel en el SNI de los p-i, para ello se dividió a los investigadores en dos grupos: en el primero, se agruparon a los candidatos a investigador(a) nacional e investigadores nacionales nivel 1 y, en el segundo, a investigadores nacionales nivel 2 y 3. Se aplicó una prueba ANOVA de un factor para verificar las diferencias entre los grupos. Los resultados de la prueba arrojaron diferencias no significativas $\left(\mathrm{F}=1.99 ; p=.160 ; \eta^{2}=.01\right)$, por lo que se acepta que no hay una diferencia en la predisposición de los p-i para realizar actividades de cooperación tecno-científica entre IES y ESP en función su nivel de SNI. En cuanto a la diferencias entre grupos de edad, la prueba ANOVA de un factor no mostró resultados significativos $\left(\mathrm{F}=3.211 ; p=.07 ; \eta^{2}=.01\right)$, por lo que se considera que la edad no tiene influencia en la percepción de llevar a cabo actividades de vinculación entre IES y ESP. Los resultados se aprecian en la tabla 3. 
Tabla 3: Comparación estadística de los grupos por variable independiente

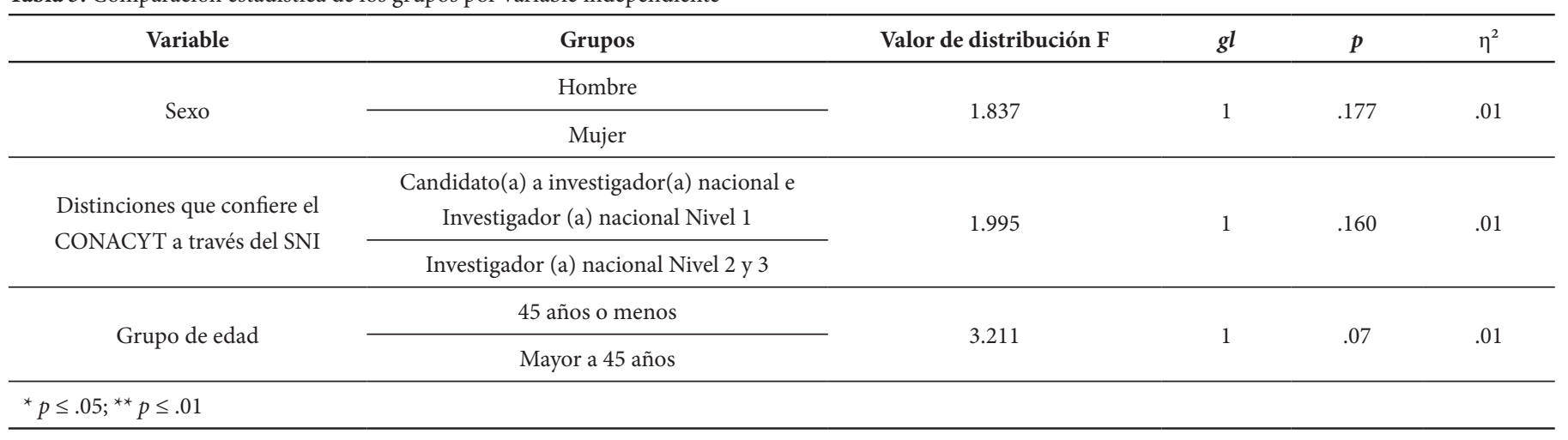

\section{Discusión y conclusiones}

Conocer la relación entre las características personales de los p-i y su actitud y predisposición hacia las actividades de cooperación tecnocientífica entre las IES y las ESP es de vital importancia para entender de mejor manera el fenómeno. Para la presente investigación, se ejecutaron distintos análisis estadísticos para la obtención de evidencias sobre la influencia de dichas características en la actitud de los p-i hacia las actividades de cooperación tecno-científica. Con el fin de que las interpretaciones de las comparaciones de las puntuaciones entre grupos sean resultado de la diferencia individual de la actitud hacia la cooperación tecnocientífica, se consideraron los hallazgos sobre invarianza factorial en función del sexo y edad presentados por Pérez-Morán et al. (2020). Debido a que en el presente estudio también se compararon grupos respecto a las distinciones que confiere el CONACYT a través del SNI, fue necesario realizar un análisis de invarianza factorial entre estos grupos. Los resultados obtenidos del AFCMG permitieron aceptar evidencias de invarianza factorial en los modelos configuracional y débil (ver tabla 2). Lo anterior, permite suponer que la estructura del modelo y la manera en la que los participantes responden a los ítems es la misma. Además, permite que las puntuaciones de los participantes puedan compararse entre grupos (Dimitrov, 2010). No obstante, los resultados en función de las distinciones del CONACYT, se deben tomar con cautela, pues, en el presente estudio, se compararon las medias de las puntuaciones de los participantes, sin la obtención de evidencias de invarianza factorial en el modelo fuerte, lo cual puede suponer un sesgo en la interpretación de los resultados (Dimitrov, 2010).

Se produjeron tres hallazgos principales sobre la relación entre las variables. Primero, se encontró que el sexo no influye en una actitud favorable hacia la realización de actividades de cooperación tecno-científica entre las IES y las ESP. La prueba ANOVA arrojó valores de diferencia no significativa y tamaño del efecto chico $(\mathrm{F}=1.84 ; p=$ $.177 ; \eta^{2}=.01$ ), por lo que se rechaza la $\mathrm{H}_{1}$. Estos resultados coinciden con las investigaciones de Bozeman y Gaughan (2011), y Haeussler y Colyvas (2011), en donde no se encontró una diferencia significativa en función del sexo del p-i. Al igual que Ding et al. (2006), se considera que los resultados del presente estudio son un reflejo de la disminución de la brecha de género en las actividades relacionadas con la cooperación tecno-científica.
Segundo, se encontró que la experiencia académica influye en la percepción favorable para realizar actividades de cooperación tecnocientífica entre IES y ESP. La comparación entre grupos arrojó valores no significativos y un tamaño del efecto chico $\left(\mathrm{F}=1.99 ; p=.160 ; \eta^{2}\right.$ $=.01$ ). Por lo anterior, se rechaza la $\mathrm{H}_{2}$, ya que se esperaba que las puntuaciones medias de los p-i con más experiencia laboral fueran significativamente mayores a los p-i con menor experiencia. Estos resultados concuerdan con los hallazgos normalmente reportados en la literatura (Perkmann et al., 2013). No obstante, hay evidencia de que los p-i Candidatos a investigadores nacionales registran significativamente más patentes en relación a los p-i con niveles 1 y 2 (Díaz-Pérez y Alarcón-Osuna, 2018). Se considera que, similar a la postura de D’Este y Fontana (2007), la reputación puede jugar un papel clave en la incursión en las actividades de cooperación tecno-científica. Asimismo, otra explicación es que la experiencia se asocia a factores determinantes para la realización de actividades de cooperación tecnocientífica, tales como capital social, mayor número de publicaciones científicas y la obtención de más apoyos gubernamentales (Perkmann et al., 2013). Por lo que se recomienda que futuros estudios exploren a profundidad el efecto de dichas variables.

Tercero, de acuerdo con los resultados del ANOVA ( $\mathrm{F}=3.211 ; p=.07$; $\eta^{2}=.01$ ), se infiere que la edad tiene un efecto no significativo y pequeño en la percepción de actividades de cooperación tecno-científica. Dado lo anterior, se rechaza la $\mathrm{H}_{3}$. Estos resultados apoyan parcialmente los hallazgos de Craig y Ponomariov (2009), y de Puerta-Sierra y Jasso (2020), en donde la edad no representa un factor determinante para la percepción de actividades de cooperación tecno-científica.

Asimismo, el presente estudio cuenta con una serie de limitaciones que deben considerarse a la hora de interpretar los hallazgos. Primero, la muestra está comprendida por $\mathrm{p}$-i de la región noroeste de México, específicamente de Baja California. Este estado cuenta con características que podrían influenciar la percepción de actividades de cooperación tecno-científica, tales como la cercanía e interacción con centros de Investigación, Desarrollo tecnológico e Innovación (I+D+i) e IES de California (López-Hernández et al., 2015). Segundo, la cantidad de participantes por grupo no es equivalente, por lo que las comparaciones pueden presentar una subrepresentación de las poblaciones aquí estudiadas. Tercero, la obtención de los datos se dió a través de un medio cuantitativo de autoinforme por lo que se 
recomienda la aplicación de métodos mixtos para contar con un análisis más integral del fenómeno, tales como entrevistas, análisis de patentes registradas por parte de los p-i y otros indicadores de cooperación tecno-científica (García-Galván, 2008).

Para futuros estudios se recomienda: (a) profundizar en el por qué los Candidatos a investigador del SNI tienen una percepción favorable para las actividades de cooperación tecno-científica en relación a los p-i con distinto nivel como investigador nacional en el SNI; (b) replicar este tipo de investigación en territorio mexicano: pese a ser campo importante, solamente se encontró una investigación que explora el tema (Puerta-Sierra y Jasso, 2020); (c) verificar si la percepción de actividades tecno-científicas varía según las características de la región donde se estudia.

\section{Referencias}

Bozeman, B., \& Gaughan, M. (2011). How do men and women differ in research collaborations? An analysis of the collaborative motives and strategies of academic researchers. Research Policy, 40, 13931402. https://doi.org/10.1016/j.respol.2011.07.002

Bozeman, B., Fay, D., \& Slade, C. (2013). Research collaboration in universities and academic entrepreneurship: The-state-of-the-art. Journal of Technology Transfer, 38, 1-67. https://doi.org/10.1007/ s10961-012-9281-8

Cabrera, M. R. (2015). De la ciudad intensiva en conocimiento a la ciudad innovadora. Aportaciones de las comunidades científicas a los ecosistemas de innovación. México: Universidad Autónoma de Baja California.

Craig, P., \& Ponomariov, B. L. (2009). University researchers working with private companies. Technovation, 29, 142-153. https://doi. org/10.1016/j.technovation.2008.03.008

Creswell, J. W. (2012). Educational Research: planning, conducting, and evaluating quantitative and qualitative research. Boston, MA: Pearson Education.

D’Este, P., \& Patel, P. (2007). University-industry linkages in the UK: What are the factors underlying the variety of interactions with industry? Research Policy, 36, 1295-1313. https://doi.org/10.1016/j.respol.2007.05.002

Diario Oficial de la Federación (2021). Acuerdo por el que se reforma el Reglamento del Sistema Nacional de Investigadores. Secretaría de Gobernación. México. http://www.dof.gob.mx/nota_detalle.php?cod igo $=5616259 \&$ fecha $=20 / 04 / 2021$

Díaz-Pérez C., \& Alarcón-Osuna M. A. (2018). Ciencia, tecnología e innovación en México: un análisis de la política pública, Estudios Regionales en Economía, Población y Desarrollo. Cuadernos de Trabajo de la UACJ, Universidad Autónoma de Ciudad Juárez, 47, 3-33. http://erevistas.uacj.mx/ojs/index.php/estudiosregionales/article/ view/2604/2428
Dimitrov, D. M. (2010). Testing for factorial invariance in the context of construct Validation. Measurement and Evaluation in Counseling and Development, 43, 121-149. https://doi.org/10.1177/0748175610373459

Ding, W. W., Murray, F., \& Stuart, T. E. (2006). Gender differences in patenting in the academic life sciences. Science, 313, 665-667. https:// doi.org/10.1126/science.1124832

Etzkowitz, H. (2003). Research groups as 'quasi-firms': the invention of the entrepreneurial university. Research Policy, 32, 109-121.

Etzkowitz, H., Webster, A., Gebhardt, C. y Cantisano, B. R. (2000). The future of the university and the university of the future: evolution of ivory tower to entrepreneurial paradigm. Research Policy, 29, 313-330.

Fichter, K., \& Tiemann, I. (2018). Factors influencing university support for sustainable entrepreneurship: Insights from explorative case studies. Journal of Cleaner Production, 175, 512-524.

Funder, D. C., \& Ozer, D. J. (2019). Evaluating Effect Size in Psychological Research: Sense and Nonsense. Advances in Methods and Practices in Psychological Science, 2, 156-168. https://doi. org/10.1177/2515245919847202

García-Galván, R. (2018). Cooperación tecnocientífica universidadempresa e interfirma: Análisis teórico integrado desde la perspectiva de la economía institucional contemporánea. Ciencia y Universidad. Revista de economía, 37, 5-30. Recuperado de http://cienciayuniversidad.com/ images/revistas/37/1cyu37\%20Rodolfo\%20Garcia\%20Galvan.pdf

García-Galván, R., Lindquist, R. \& Morales, M. (2021). La colaboración de las organizaciones públicas del conocimiento con las empresas: una mirada de empresarios. Paradigma Económico, 13, 147-180. https://paradigmaeconomico.uaemex.mx/article/view/14878/11906

Giuliani, E., Morrison, A., Pietrobelli, C., \& Rabellotti, R. (2010). Who are the researchers that are collaborating with industry? An analysis of the wine sectors in Chile, South Africa and Italy. Research Policy, 39, 748-761. https://doi.org/10.1016/j.respol.2010.03.007

Haeussler, C., \& Colyvas, J. A. (2011). Breaking the Ivory Tower: Academic entrepreneurship in the life sciences in UK and Germany. Research Policy, 40, 41-54. https://doi.org/10.1016/j.respol.2010.09.012

Hair, J. F., Black, W. C., Babin, B. J. \& Anderson R. E. (2019). Multivariate Data Analysis. Cengage Learning.

Hu, L. T., \& Bentler, P. M. (1999). Cutoff criteria for fit indexes in covariance structure analysis: Conventional criteria versus new alternatives. Structural Equation Modeling, 6, 1-55. https://doi. org/10.1080/10705519909540118

López-Hernández, C. G., Plascencia-López, I., \& Alcalá-Álvarez, M. C. (2015). Ecosistema de Innovación Binacional en el Noroeste de México Caso de Estudio Baja California-California [Sesión de conferencia]. XVI Congreso Latino-Iberoamericano de Gestión Tecnológica, Porto Alegre, RGS, Brasil. http://altec2015.nitec.co/altec/papers/253.pdf 
López-Leyva, S. (2002). La vinculación y los investigadores. Perfiles Educativos, 24. http://www.redalyc.org/pdf/132/13209806.pdf

Morales, M,. \& Rodríguez, J. C. (2021). Percepción de los investigadores de Baja California sobre la cooperación tecnocientífica que se realiza en las instituciones de educación superior. En R. GarcíaGalván, J. C. Rodríguez y A. A. Chaparro (Coords.). Cooperación de las organizaciones del conocimiento con el entorno productivo y social de Baja California. Ed. Qartuppi. Qartuppi. http://doi.org/10.29410/ QTP.21.02

Morales, M. (2019). Cooperación Tecnocientífica IES-Sector Productivo desde la perspectiva del cambio institucional. Evidencias de Baja California (Tesis de doctorado). Universidad Autónoma de Baja California. http://iide.ens.uabc.mx/documentos/divulgacion/tesis/ DCE/2016/Marcela_Morales_Paez.pdf

Morales, M., \& García-Galván, R. (2020). Colaboración tecnocientífica academia-empresa. Un análisis de la percepción de profesores-investigadores. Revista Economía, Teoría y Práctica, 28, 173-208. https://economiatyp.uam.mx/index.php/ETYP/article/ view/460
Pérez-Moran, J. C., Morales-Páez, M., \& Bernal-Baldenebro, B. (2021). Propiedades psicométricas de un cuestionario para medir cooperación tecnocientífica entre universidades y sector productivo [Manuscrito presentado para su publicación]. Instituto de Investigación y Desarrollo Educativo, Universidad Autónoma de Baja California.

Perkmann, M., Tartari, V., McKelvey, M., Autio, E., Broström, A., D'Este, P., ... \& Sobrero, M. (2013). Academic engagement and commercialisation: A review of the literature on university-industry relations. Research Policy, 42, 423-442. https://doi.org/10.1016/j.respol.2012.09.007

Puerta-Sierra, L., \& Jasso, J. (2020). University-Industry Collaboration. An Exploration of An Entrepreneurial University in Mexico. Journal of Technology Management \& Innovation, 5(3), 33-39. https:// www.jotmi.org/index.php/GT/article/view/3381/1303

Stuart, T. E., \& Ding, W. W. (2006). When do scientists become entrepreneurs? The social structural antecedents of commercial activity in the academic life sciences. American Journal of Sociology. https://doi. org/10.1086/502691

Tartari, V., \& Salter, A. (2015). The engagement gap: Exploring gender differences in University - Industry collaboration activities. Research Policy, 44, 1176-1191. https://doi.org/10.1016/j.respol.2015.01.014 